\title{
AN EFFICIENT HOMOGENIZATION METHOD USING THE TRIGONOMETRIC INTERPOLATION AND THE FAST FOURIER TRANSFORM
}

\author{
Ngoc-Trung Nguyen ${ }^{1}$, Christian Licht ${ }^{2}$ and Jin-Hwe Kweon ${ }^{3}$ \\ ${ }^{1}$ Kangwon National University, Chunchon, Kangwon-do, South Korea \\ ${ }^{2}$ LMGC, Université de Montpéllier 2, France \\ ${ }^{3}$ School of Mechanical and Aerospace Engineering \\ Gyeongsang National University, South Korea
}

\begin{abstract}
This study focuses on formulation of the Augmented Lagrangian and application of the Uzawa's algorithm to solve the homogenization problem of microscopic periodic media as in composites. Unlike in the finite element model, an equally spaced grid system associated with the microstructure domain is used instead of a finite element mesh topology. Moreover, the trigonometric interpolations for the field variables at every grid point help to handle the periodic conditions. The proposed approach is a compromise between Lagrange multiplier and penalty methods, in that it enables exact representation of constraints while using penalty terms to facilitate the iteration procedure. A typical homogenization problem will be solved using this approach. The results show good consistency with those in literatures. Effects of the grid density and the penalty parameter on the convergence have also been investigated.
\end{abstract}

Keywords: Homogenization, Augmented Lagrangian method, trigonometric interpolation.

\section{INTRODUCTION}

For composites of complex microstructures, there are two different scales associated with microscopic and macroscopic behaviors to deal with: the slowly varying global variables and the rapidly oscillating local variables. To model a structure of such kind of material using the finite element method (FEM) one should utilize very fine mesh density so that the details at the microscale size can be captured. That leads to a very high computational cost and sometimes it is impossible to perform the analysis due to extremely high requirements of computer resources. Instead, a process so-called homogenization is used to characterize the heterogeneous material as a homogenized one and the equivalent material properties are then used in the simulation of the whole structure as in a regular FEM analysis.

For the sake of simplicity, the materials can be considered as an assembled body of periodic unit cells as shown in Fig. 1. The assumption of such periodic media has been widely used to develop in both the mathematical analysis and the numerical models $[1,12]$. 
Among various approaches to predict the effective properties of composites, the mathematical homogenization method is preferable due to its systematic background and the ease to implement $[1,10,11]$. One may use a FEM program with a slight modification to solve the homogenization problem to obtain the homogenized coefficients without difficulty $[4,5,9]$. Note that the periodicity conditions must be imposed through constraint equations to reflect the repeatability of the microstructure. Evaluation of several treatments of periodicity was discussed in [2]. However, using FEM to solve the homogenization problem may have limitation in meshing the complicated microstructures and handling with a large number of degree of freedom (DOF) in analysis [8].
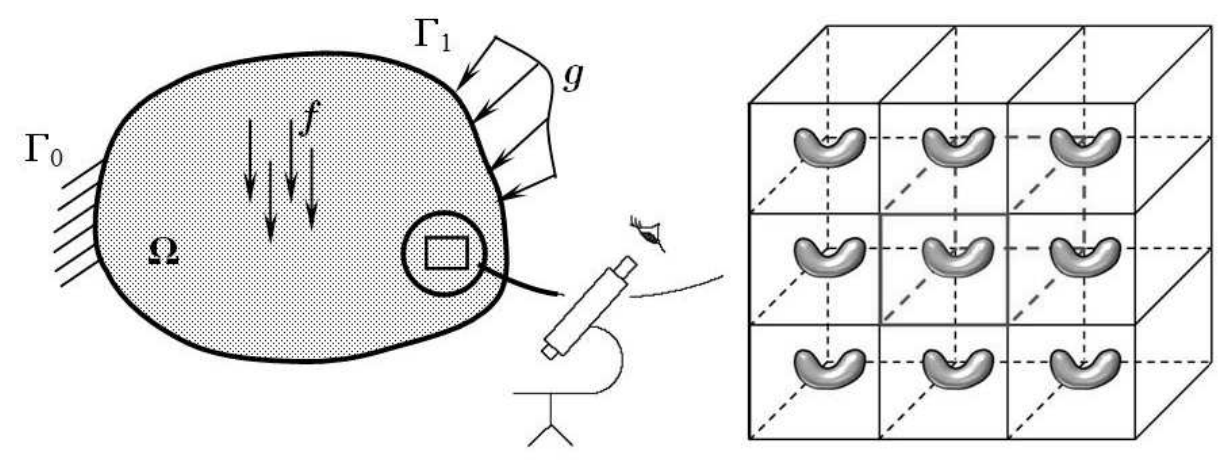

Fig. 1. The macroscale and microscale of the homogenization problem

The objective of this study is to develop an effective approach to solve the homogenization problem. The variable decomposition technique [3] together with formulation of an Augmented Lagrangian is used leading to a saddle-point problem. The Uzawa's algorithm [6] is then applied to solve the saddle-point problem by iterations. This method is reliable due to the fact that it is proved to be converged [3]. To eliminate the condition on periodicity of the field variables, we employ the trigonometric interpolations. The field variables will then automatically satisfy the periodicity conditions. A grid system associated with the microstructure domain is utilized in this approach instead of a mesh configuration as in the finite element analysis. The meshing and the handling large number of degrees of freedom issues will not be encountered regardless the complexity of the composite microstructure.

\section{HOMOGENIZATION OF PERIODIC MEDIA}

From the asymptotic expansion $[1,11]$, the homogenized elasticity tensor can be determined explicitly by:

$$
a_{i j k h}^{\text {hom }}=\frac{1}{|\mathbf{Y}|} \int_{\mathbf{Y}}\left(a_{i j k h}(\mathbf{y})+a_{i j r s}(\mathbf{y}) e_{r s}\left(\chi^{k h}\right)\right) d \mathbf{y}
$$

where $\mathbf{Y}=\left\{\mathbf{y} \in \Re^{n}, 0 \leq y_{i} \leq Y_{i}, i=\overline{1, n}\right\}$,

$|\mathbf{Y}|$ is the volume of the unit cell, 
$\langle\bullet\rangle=\frac{1}{|\mathbf{Y}|} \int_{\mathbf{Y}}(\bullet) d \mathbf{y}$ denotes the average over the unit cell $\mathbf{Y}$,

$\chi^{k h}$ in (1) is the solution of the cell problem:

$$
\left\{\begin{array}{l}
-\frac{\partial}{\partial y_{j}} a_{i j r s}(\mathbf{y}) e_{r s}\left(\chi^{k h}\right)=\frac{\partial}{\partial y_{j}} a_{i j k h}(\mathbf{y}) \\
\chi^{k h} \text { is } \mathbf{Y}-\text { periodic }
\end{array}\right.
$$

with the periodicity condition defined by:

If $\mathbf{y} \in \mathbf{Y} \mapsto v_{i}(\mathbf{y}): \mathbf{Y}$ - periodic or $v_{i}(y) \in V_{\text {per }}(\mathbf{Y})$ then $v_{i}(\mathbf{y})$ takes equal values on the opposite faces of $\mathbf{Y}$.

Generally, we can obtain the solution $\mathbf{v}_{\mathbf{E}}=\chi^{k h}$ by solving the six cell problems and then compute the homogenized elasticity coefficients according to (1).

As an alternative, the homogenization problem (2) with periodicity conditions can be formulated in the following forms:

For a given macroscopic strain $\mathbf{E}$,

$$
\left(\mathbf{P}_{\mathbf{E}}\right)\left\{\text { Find } \mathbf{v}_{\mathbf{E}} \in V_{\text {per }}(\mathbf{Y}) \text { such that } \mathbf{J}_{\mathbf{E}}\left(\mathbf{v}_{\mathbf{E}}\right) \leq \mathbf{J}_{\mathbf{E}}(\mathbf{v}), \forall \mathbf{v} \in V_{\text {per }}(\mathbf{Y})\right\}
$$

where $\mathbf{J}_{\mathbf{E}}(\mathbf{v})=\frac{1}{2|\mathbf{Y}|} \int_{\mathbf{Y}} \mathbf{a}(\mathbf{E}+e(\mathbf{v}))(\mathbf{E}+e(\mathbf{v})) d \mathbf{y}$

Note that the problems (2) and (3) are equivalent. Moreover, the variational formulation (3) is equivalent to a problem of minimization with constraints:

$$
\left\{\begin{array}{l}
\text { Find } \mathbf{v} \in V_{\text {per }}(\mathbf{Y}) \text { such that } \mathbf{J}(\mathbf{v}) \rightarrow \min \\
\mathbf{J}(\mathbf{v})=\frac{1}{2} \mathbf{a}(\mathbf{v}, \mathbf{v})-l(\mathbf{v})
\end{array}\right.
$$

where $\mathbf{a}(\mathbf{v}, \mathbf{v})=\frac{1}{|\mathbf{Y}|} \int_{\mathbf{Y}} \mathbf{a e}\left(\mathbf{v}_{\mathbf{E}}\right) \mathbf{e}(\mathbf{v}) d \mathbf{y}, l(\mathbf{v})=-\frac{1}{|\mathbf{Y}|} \int_{\mathbf{Y}} \mathbf{a E e}(\mathbf{v}) d \mathbf{y}$

Again, by solving 6 problems of formulation (3) or (4) with the imposed macroscopic strains $E_{i j}=\left(T^{k h}\right)_{i j}=\frac{1}{2}\left(\delta_{i k} \delta_{j h}+\delta_{i h} \delta_{j k}\right)$, where $\delta_{i j}$ is the Kronecker delta symbol; the homogenized coefficients are determined by:

$$
a_{i j k h}^{\text {hom }}=\left\langle\sigma_{i j}^{k h}\right\rangle=\frac{1}{|\mathbf{Y}|} \int_{\mathbf{Y}} \sigma_{i j}^{k h} d \mathbf{y}=\frac{1}{|\mathbf{Y}|} \int_{\mathbf{Y}} \mathbf{a}\left(\mathbf{T}^{k h}+e\left(\mathbf{v}_{\mathbf{T}^{k h}}\right)\right) d \mathbf{y}
$$

In the following sections, we will briefly review the trigonometric interpolation technique and the discrete Fourier transforms. Then, we reformulate the minimization problem (5) by using the Augmented Lagrangian method and the variable decomposition technique. Based on these variational equations, an in-house code will be developed.

\section{TRIGONOMETRIC INTERPOLATION AND THE DISCRETE FOURIER TRANSFORMATIONS}

Consider a unit cell $\mathbf{Y}=] 0,1\left[^{3}\right.$, with a given positive number $n$ we define the grid points: $\left(x_{j}, y_{k}, z_{l}\right)=\left(\frac{j}{n}, \frac{k}{n}, \frac{l}{n}\right)$, where $j, k, l \in\{0,1, \ldots, n-1\}$ as shown in Fig. 2. 


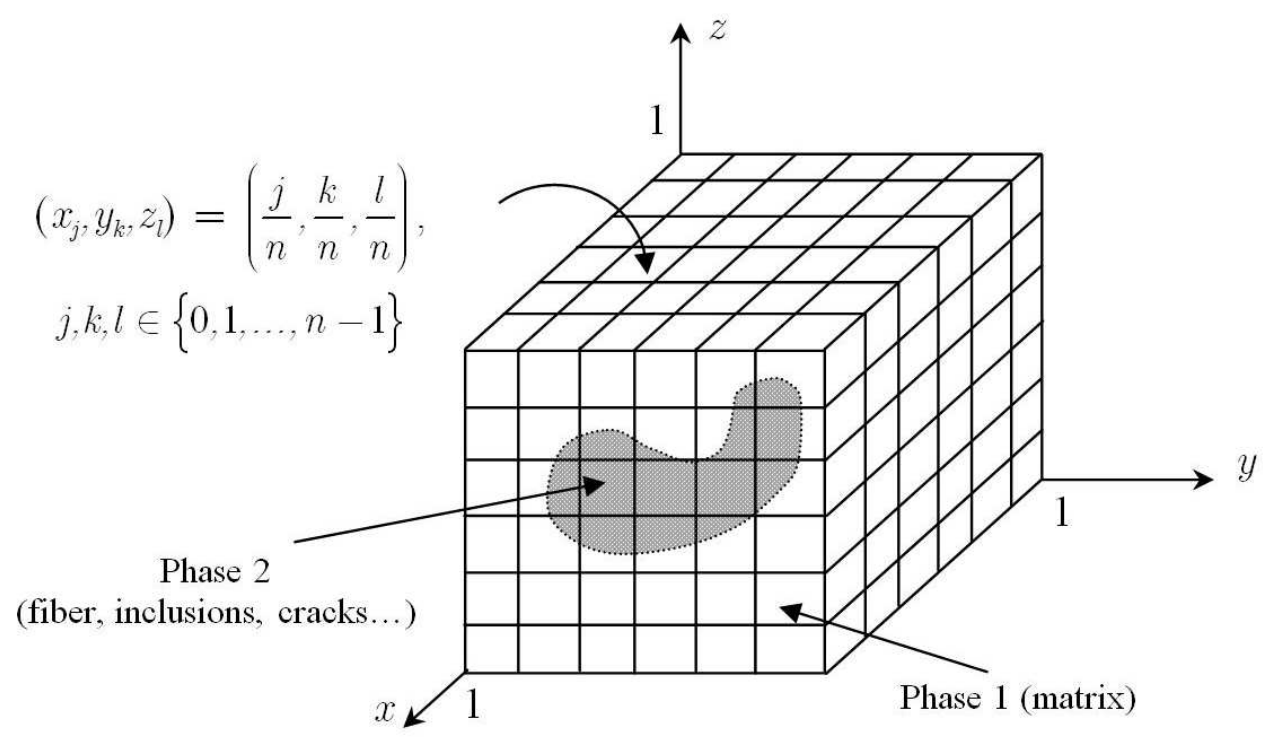

Fig. 2. Grid system of the periodic unit cell

Due to the periodicity of the field variables, the trigonometric interpolation of a function $f(x, y, z)$ is defined through the values at grid points $f\left(x_{j}, y_{k}, z_{l}\right)$ in the system by using the inverse discrete Fourier transform:

$\hat{f}\left(x_{j}, y_{k}, z_{l}\right)=\sum_{r, s, t} \frac{\hat{f}_{j k l}}{n^{3}} w_{j k l}^{-1}\left(x_{r}, y_{s}, z_{t}\right)=\sum_{r, s, t} \frac{\hat{f}_{j k l}}{n^{3}} e^{2 i \pi j x_{r}} e^{2 i \pi k y_{s}} e^{2 i \pi l z_{t}}, r, s, t \in\{0,1, \ldots, n-1\}$

where, the coefficients $\hat{f}_{j k l}$ of the interpolated function $\hat{f}(x, y, z)$ are determined by the discrete Fourier transform:

$$
\hat{f}_{j k l}=\sum_{r, s, t} f\left(x_{r}, y_{s}, z_{t}\right) w_{j k l}\left(x_{r}, y_{s}, z_{t}\right)=\sum_{r, s, t} f\left(x_{r}, y_{s}, z_{t}\right) e^{-2 i \pi j x_{r}} e^{-2 i \pi k y_{s}} e^{-2 i \pi l z_{t}}
$$

The values of the interpolated function $\hat{f}(x, y, z)$ should be equal to the given values of the function $f(x, y, z)$ at grid points:

$$
\hat{f}\left(x_{j}, y_{k}, z_{l}\right)=f\left(x_{j}, y_{k}, z_{l}\right), \forall j, k, l \in\{0,1, \ldots, n-1\}
$$

With the above definition of trigonometric interpolation, the field variables in the homogenization problem will then automatically satisfy the periodicity conditions. This benefit helps to eliminate constraints on variables and, as a consequence, to reduce the complexity of the problem.

\section{THE AUGMENTED LAGRANGIAN FORMULATION}

By introducing a supplementary variable $\mathbf{q}$, linked to $\mathbf{v}$ through the relation $\mathbf{q}=\nabla \mathbf{v}$, the problem (4) can be reformulated in the following form: 
For a given macroscopic strain $\mathbf{E}$, find

$$
\min \left\{\frac{1}{|\mathbf{Y}|} \int_{\mathbf{Y}} \frac{1}{2} \mathbf{a}\left(\mathbf{E}+\mathbf{q}_{s}\right) \cdot\left(\mathbf{E}+\mathbf{q}_{s}\right) d \mathbf{y}, \forall \mathbf{v} \in V_{\text {per }}(\mathbf{Y}) \text { and } \mathbf{q}=\nabla \mathbf{v}\right\}
$$

where $\mathbf{q}_{\mathbf{s}}=\frac{1}{2}\left(\mathbf{q}+\mathbf{q}^{\mathrm{T}}\right)$ is the symmetric part of the tensor $\mathbf{q}=\nabla \mathbf{v}$. The problem (6) is a minimization problem in $\{\mathbf{q}, \mathbf{v}\}$ with the supplementary constraint $\mathbf{q}=\nabla \mathbf{v}$. To handle this constraint we use a Largrange multiplier to reduce the problem (6) (and also the problems (2), (3) and (4)) to a saddle-point problem:

$$
L(\mathbf{v}, \mathbf{q}, \mu)=\frac{1}{|\mathbf{Y}|} \int_{\mathbf{Y}} \frac{1}{2} \mathbf{a}\left(\mathbf{E}+\mathbf{q}_{s}\right)\left(\mathbf{E}+\mathbf{q}_{s}\right) d \mathbf{y}+\frac{1}{|\mathbf{Y}|} \int_{\mathbf{Y}} \mu(\nabla \mathbf{v}-\mathbf{q}) d \mathbf{y}
$$

where $\mu$ is a Lagrange multiplier. For $r \geq 0$, the Augmented Lagrangian is defined by:

$$
L_{r}(\mathbf{v}, \mathbf{q}, \mu)=\frac{1}{|\mathbf{Y}|} \int_{\mathbf{Y}} \frac{\mathbf{a}}{2}\left(\mathbf{E}+\mathbf{q}_{s}\right)\left(\mathbf{E}+\mathbf{q}_{s}\right) d \mathbf{y}+\frac{1}{|\mathbf{Y}|} \int_{\mathbf{Y}} \mu(\nabla \mathbf{v}-\mathbf{q}) d \mathbf{y}+\int_{\mathbf{Y}} \frac{r}{2}|\nabla \mathbf{v}-\mathbf{q}|^{2} d \mathbf{y}
$$

Then, the problem (7) is equivalent to:

Find the saddle-point $(\mathbf{u}, \mathbf{p}, \lambda)$ such that:

$$
L_{r}(\mathbf{u}, \mathbf{p}, \lambda)=\min _{\mathbf{v}, \mathbf{q}} \max _{\mu} L_{r}(\mathbf{v}, \mathbf{q}, \mu)
$$

Note that if $\mathbf{v}_{\mathbf{E}}$ is the solution of the problem (3) and (4) then it is equivalent to the fact that $\left\{\mathbf{v}_{\mathbf{E}}, \mathbf{q}_{\mathbf{E}}=\nabla \mathbf{v}_{\mathbf{E}}, \lambda\right\}$ is a saddle-point of $L_{r}$. Thus, we have:

$$
L_{r}\left(\mathbf{v}_{\mathbf{E}}, \mathbf{q} \mathbf{E}, \mu\right) \leq L_{r}\left(\mathbf{v}_{\mathbf{E}}, \mathbf{q}_{\mathbf{E}}, \lambda\right) \leq L_{r}(\mathbf{v}, \mathbf{q}, \lambda), \forall\{\mathbf{v}, \mathbf{q}, \mu\}
$$

Consequently, instead of solving the problem (3) or (4) for $\mathbf{v}_{\mathbf{E}}$, we aim to find the saddle-point $\left\{\mathbf{v}_{\mathbf{E}}, \mathbf{q}_{\mathbf{E}}, \lambda\right\}$ of $L_{r}$ by using the so-called Uzawa's algorithm $[3,6]$. The threestep process at each iteration and the details of the method are given below:

Initialization: $\left\{\mathbf{q}_{\mathbf{E}}^{0}, \lambda^{1}\right\}$ are given arbitrarily.

With values of $\left\{\mathbf{q}_{\mathbf{E}}^{n-1}, \lambda^{n}\right\}$ calculated at the $n$-th iteration, $\mathbf{v}_{\mathbf{E}}^{n}, \mathbf{q}_{\mathbf{E}}^{n}$ and $\lambda^{n+1}$ at the $(n+1)$-th iteration are to be determined successively by:

STEP 1: Minimization of $L_{r}$ in $\mathbf{v}$

Taking the variation of $L_{r}$ in $\mathbf{v}$, the condition of minimization leads to:

$$
\int_{Y}\left[\left(-\lambda^{n} \nabla \psi\right)+\left(-r \mathbf{q}_{\mathbf{E}}^{n-1} \nabla \psi+r \nabla \mathbf{v}_{\mathbf{E}}^{n} \nabla \psi\right)\right] d \mathbf{y}=0, \forall \psi \in V_{p e r}(\mathbf{Y})
$$
yield:

By applying the divergence theorem and the periodicity condition of $\psi$ to (9), we

STEP 2: Minimization of $L_{r}$ in $\mathbf{q}$

$$
\Delta \mathbf{v}_{\mathbf{E}}^{n}=\operatorname{div}\left(\mathbf{q}_{\mathbf{E}}^{n-1}+\frac{\lambda^{n}}{r}\right)
$$

Taking the variation of $L_{r}$ in $\mathbf{q}$, the condition of minimization leads to:

$$
\mathbf{a}\left(\mathbf{q}_{\mathbf{E}}^{n}\right)_{\mathbf{s}}+r \mathbf{q}_{\mathbf{E}}^{n}=-\mathbf{a E}+r \nabla \mathbf{v}_{\mathbf{E}}^{n}-\lambda^{n}
$$


By solving (10) we can obtain the symmetric and the anti-symmetric parts of $\mathbf{q}_{\mathbf{E}}$. The updated values of $\mathbf{q}_{\mathbf{E}}^{n}$ are: $\mathbf{q}_{\mathbf{E}}^{n}=\left(\mathbf{q}_{\mathbf{E}}^{n}\right)_{s}+\left(\mathbf{q}_{\mathbf{E}}^{n}\right)_{a}$

STEP 3: Updating $\lambda^{n}$

$$
\lambda^{n+1}=\lambda^{n}+r\left(\nabla \mathbf{v}_{\mathbf{E}}^{n}-\mathbf{q}_{\mathbf{E}}^{n}\right)
$$

The solutions of (8) are obtained by iterations. For each iteration, there are three steps, in which (9), (10) and (11) are readily to be processed by using the above-mentioned trigonometric interpolations and the Fourier transforms of displacement field variables. To avoid obtaining a local minimizer solution, the convergence criteria is employed such that the iterating process terminates when the relative error of a variable is smaller than a chosen value $\varepsilon$ for a NCONV number of successive iteration. The relative error is taken in $L^{2}-$ norm, i.e. $\left\|u_{i}^{k+1}-u_{i}^{k}\right\|_{L^{2}} /\left\|u_{i}^{k}\right\|_{L^{2}} \leq \varepsilon$. The variable value $u_{i}$ can be the displacement at grid points $\mathbf{v}$, divergence of the displacement $\mathbf{q}$ or the Lagrange multiplier $\lambda$. Once convergence has been reached, $\mathbf{q}_{\mathbf{E}}$ coincides with $\nabla \mathbf{v}_{\mathbf{E}}$. The Uzawa's algorithm with a suitable choice of the initial and the multiplier values has been proved to achieve better convergence.

\section{NUMERICAL EXAMPLE}

To verify the proposed method, we investigate a typical example of a unidirectional fiber-reinforced composite material using $2 \mathrm{D}$ model to compare with results in [7].

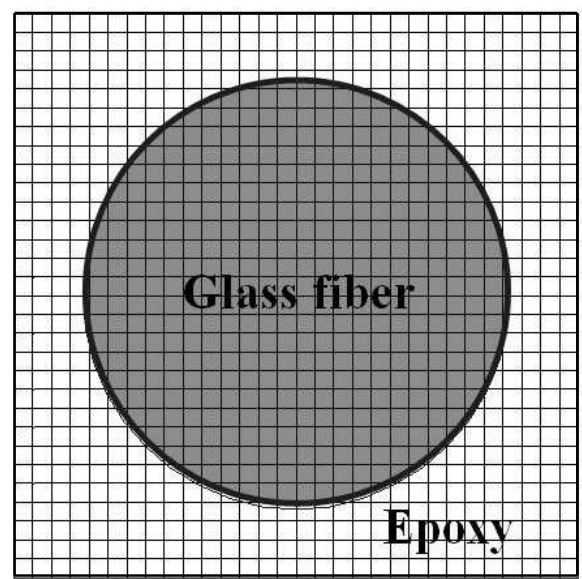

Fig. 3. Grid system of the unit cell

A grid system of the unit cell for the 2D model is used to predict the properties of the glans fiber/epoxy reinforced composite system as shown in Fig. 3. The volume fraction of fibers is $45 \%$. The plane strain and perfect bonding between constituents assumptions are made. The data on Young's modulus and Poisson's ratio of glass fiber and epoxy resin are given in Table 1.

Note that for a $2 \mathrm{D}$ problem, we use an exchange in notation between the tensor $a_{i j k h}$ and its matrix form $C_{i j}$. Explicitly, in this problem we denote: $C_{11}=a_{1111}, C_{22}=a_{2222}$, 
Table 1. Material properties of glass fiber and epoxy

\begin{tabular}{|l|c|c|}
\hline Material & $\mathrm{E}(\mathrm{GPa})$ & $\nu$ \\
\hline E-glass fibers & 70 & 0.2 \\
\hline Epoxy resin & 3.5 & 0.35 \\
\hline
\end{tabular}

$C_{12}=a_{1122}$ and $C_{66}=a_{1212}$. To obtain the homogenized modulus, we will solve 3 cell problems by successively impose the three macroscopic strains:

$$
\mathbf{E}=\left(\mathbf{T}^{11}\right)=\left[\begin{array}{ll}
1 & 0 \\
0 & 0
\end{array}\right], \mathbf{E}=\left(\mathbf{T}^{22}\right)=\left[\begin{array}{ll}
0 & 0 \\
0 & 1
\end{array}\right] \text { and } \mathbf{E}=\left(2 \mathbf{T}^{12}\right)=\left[\begin{array}{ll}
0 & 1 \\
1 & 0
\end{array}\right]
$$
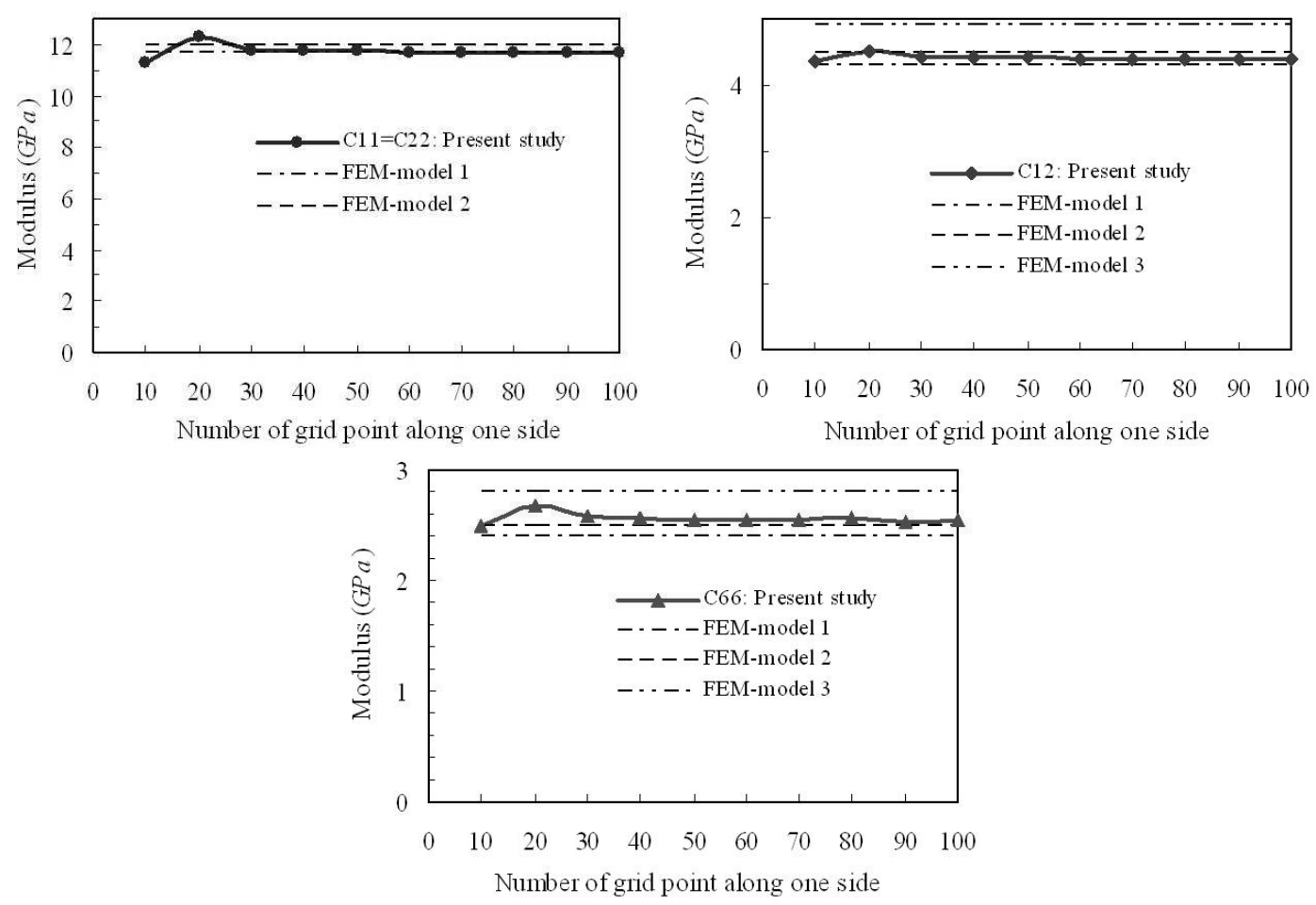

Fig. 4. Comparison between the results obtained by the present study and those of three FEM models in [7]: (a) $C_{11}=C_{22}$ coefficients, (b) $C_{12}=C_{21}$ coefficients,

(c) $C_{66}$ coefficient

Calculations of the homogenized values and comparisons with [7] are shown in Fig. 4. A quite good agreement can be observed. Note that [7] used the finite element method to solve the problem with different geometries and sizes of the unit cell. The obtained results of the present study also closely match with those in [9]. With a coarser grid density, the solutions show a small discrepancy with the referred values. However, for finer grid density the solutions are stable and almost identical to those of [7]. The sensitiveness of the solution on the grid density is thus negligible after a certain level. Hence, to employ 
the developed program efficiently we can use a rather coarse grid system to obtain results with the same accuracy as in case of fine grid resolution.

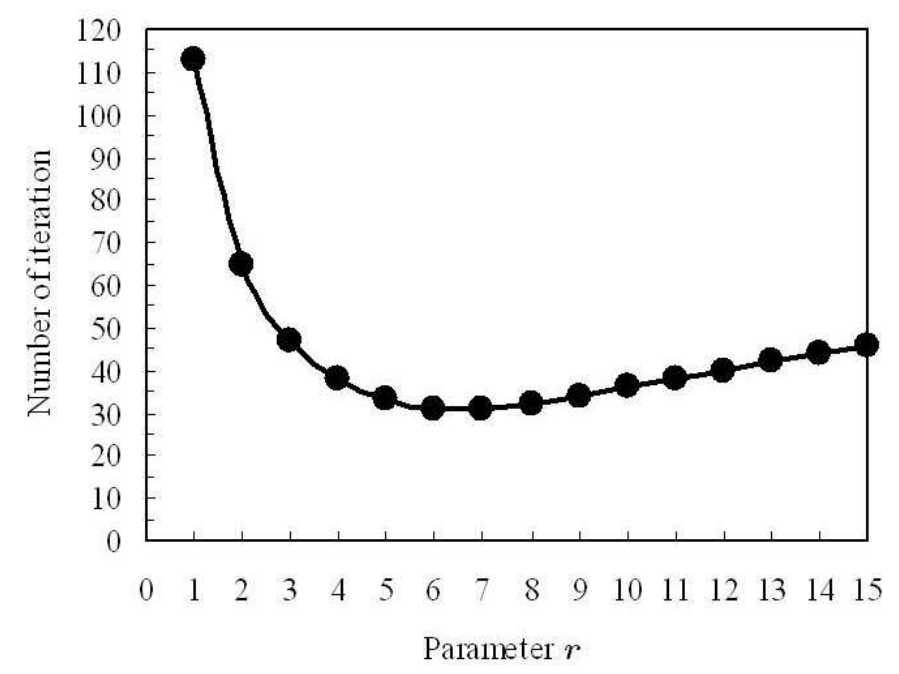

Fig. 5. Effects of the penalty parameter $r$ on the convergence

The influence of the parameter $r$ on the convergence is shown in Fig. 5. We may find that the penalty parameter needs not to tend to infinity to obtain the exact solution as in the ordinary penalization methods, it can be small. That is one of the advantages of the Augmented Lagrangian method, appearance of the last term in (7) improves the convergence properties of the algorithm.

\section{CONCLUSION}

The Augmented Lagrangian approach associated with the trigonometric interpolation in this study shows its effectiveness in solving the homogenization problems. The trigonometric interpolation helps to remove the constraint on periodicity. Furthermore, the Augmented Lagrangian method is a compromise between Lagrange multiplier and penalty methods, in that it enables exact representation of constraints while using penalty terms to facilitate the iterative procedure. Combination of these two advantages leads to a quite promising and potential way for handling the homogenization problems, especially for predicting properties of very complicated microstructured materials. Besides, the concept in this approach makes it ready to be used with a microscope image of the real microstructures. The meshing and the handling large number of DOF issues will not be encountered because the grid system is used instead of a topology of the finite element nodes. The applicability of the algorithm discussed in this study is broad, ranging from linear elasticity to nonlinear and/or plasticity applications. 
An efficient homogenization method using the trigonometric interpolation and the fast fourier transform 223

\section{REFERENCES}

[1] A. Bensoussan, J. L. Lions, G. Papanicolaou, Asymptotic analysis for periodic structures, North-Holland Publishing Company, (1978).

[2] O. Debordes, C. Licht, J. J. Marigo, P. Mialon, J. C. Michel, P. Suquet, Charges limites de milieux fortement heterogenes, Note Technique No. 85-3, Laboratoire de Mecanique Generale des Milieux Continus, Universite des Sciences et Techniques du Languedoc, (1985).

[3] M. Fortin, R. Glowinski (Eds.), Augmented Lagrangian Methods: Applications to the Numerical Solution of Boundary-Value Problems, North-Holland Publishing Company, (1982).

[4] J. M. Guedes, N. Kikuchi, Preprocessing and postprocessing for materials based on the homogenization method with adaptive finite element methods, Computer Methods in Applied Mechanics and Engineering, 83(2) (1990), 143 - 198.

[5] B. Hassani, A direct method to derive the boundary conditions of the homogenization equation for symmetric cells, Communications in Numerical Methods in Engineering, 12(3) (1996), 185 $-196$.

[6] C. Licht, Frottement, Viscoplasticité et Homogénéisation, PhD Thesis, University Montpéllier II, (1987).

[7] D. Lukkassen, L. E. Persson, P. Wall, Some engineering and mathematical aspects on the homegenization method, Composites Engineering, 5(5) (1995).

[8] H. Moulinec, P. Suquet, A numerical method for computing the overall response of nonlinear composites with complex microstructure, Computer Methods in Applied Mechanics and Engineering, 157(1-2) (1998), 69 - 94.

[9] N. T. Nguyen, K. H. Nguyen, Y. B. Park, J. H. Kweon, Characterization of composite materials with complex microstructure, Proceeding of the KSAS conference, Gyeong-ju, South Korea, (2009).

[10] O. A. Oleinik, A. S. Shamaev, G. A. Yosifian, Mathematical problems in elasticity and homogenization, North-Holland, (1992).

[11] E. Sanchez-Palencia, A. Zaoui (Eds.), Homogenization Techniques for Composite Media, Springer, (1987).

[12] L. Tartar, Quelques remarques sur l'homogénéisation, in: Fujita (Ed.), Functional analysis and numerical analysis, Japan Society for the Promotion of Science, Tokyo, (1978).

Received June 20, 2011 